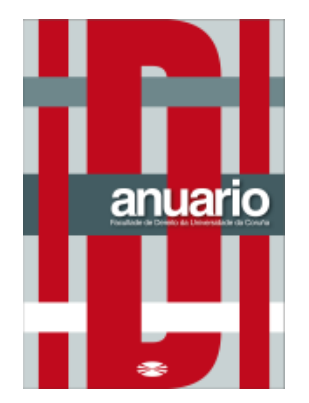

Anuario da Facultade de Dereito da Universidade da Coruña

Vol. 20 (2016), pp. 117-125

ISSNe: 2530-6324 || ISSN: 1138-039X

DOI: https://doi.org/10.17979/afdudc.2016.20.0.1916

\title{
LA FLEXIBILIZACIÓN DE LA JORNADA DE TRABAJO EN ESPAÑA. INFORME DESDE LA ÓPTICA DEL DERECHO SOCIAL EUROPEO*
}

\author{
JESÚS MARTÍNEZ GIRÓN \\ Catedrático de Derecho del Trabajo \\ Facultad de Derecho. Universidad de A Coruña \\ AlBerto ARUfe VARELA \\ Catedrático acreditado de Derecho del Trabajo \\ Facultad de Derecho. Universidad de A Coruña
}

Fecha de recepción: 30.6.2016

Fecha de aceptación: 15.7.2016

Resumen: Tras las últimas reformas laborales habidas en España, este informe analiza - desde el punto de vista del Derecho social de la Unión Europea - la puesta en práctica de una jornada de trabajo más flexible en nuestro país, sobre la base de una muestra significativa de convenios colectivos recientes de ámbito estatal publicados al amparo de dichas reformas.

Palabras clave: España, Flexibilización, Jornada de trabajo, Negociación colectiva, Reformas laborales

\begin{abstract}
After the last labor reforms we had in Spain, this report analyses - from the viewpoint of social law of the European Union - the implementation of a more flexible working time in our country, on the basis of a significant sample of recent and nationwide collective bargaining agreements published under such reforms.
\end{abstract}

Keywords: Spain, Flexibility, Working time, Collective bargaining, Labor reforms

\footnotetext{
* Ponencia defendida en el «VII. Internationaler Arbeitsrechtlicher Dialog (IAD) zum Thema "Arbeitszeiten"», celebrado en la Facultad de Derecho de la Universidad de St. Gallen (Suiza), los días 30 y 31 octubre 2014, organizado por el Forschungsinstitut für Arbeit und Arbeitsrecht, de dicha Universidad.
} 
SUMARIO: I. PRINCIPIOS BÁSICOS Y ESTRUCTURA. II. APLICACIÓN PRÁCTICA. III. HECHOS Y CIFRAS. IV. PROSPECTIVA

\section{PRINCIPIOS BÁSICOS Y ESTRUCTURA}

1. Como es lógico, la regulación legal española de la jornada está totalmente condicionada por el Derecho de la Unión Europea sobre el tema. Este Derecho comunitario está actualmente contenido en la Directiva 2003/88/CE del Parlamento Europeo y del Consejo, de 4 noviembre 2003, relativa a determinados aspectos de la ordenación del tiempo de trabajo. En lo esencial, esta Directiva aparece transpuesta al ordenamiento interno español por los artículo 34 a 38 del Estatuto de los Trabajadores de 1995, que es la norma laboral española más importante ${ }^{1}$. El Reino de España ha sido condenado una vez por el Tribunal de Justicia de la Unión Europea, por causa de la defectuosa transposición de dicha Directiva al ordenamiento interno español. Es la Sentencia de 20 mayo 2010, en el asunto Comisión Europea contra Reino de España ${ }^{2}$, que declara que el ordenamiento jurídico español no había previsto la transposición «por lo que respecta al personal no civil de las Administraciones públicas», esto es, al personal militar. La explicación de esta condena es consecuencia del hecho de que los artículos 34 a 38 del Estatuto de los Trabajadores se aplican sólo a los trabajadores, tanto del sector público como del sector privado, pero no a los funcionarios públicos. La jornada de los funcionarios se regula en otra Ley diferente, que es el Estatuto Básico del Empleado Público de $2007^{3}$. Los artículos 34 a 38 del Estatuto de los Trabajadores regulan la jornada ordinaria de trabajo, pero no las jornadas especiales (reducidas o ampliadas). Estas jornadas especiales están reguladas en un reglamento de desarrollo del Estatuto de los Trabajadores, que fue aprobado por Real Decreto 1561/1995, de 21 septiembre.

2. Según los artículos 34 a 38 del Estatuto de los Trabajadores, en España hay tres jornadas ordinarias de trabajo distintas, que son la jornada diaria, la jornada semanal y la jornada anual. Dichos artículos del Estatuto de los Trabajadores delimitan estas tres jornadas ordinarias de trabajo utilizando dos criterios distintos, respectivamente relativos a cuántas horas hay que trabajar (criterio positivo) y a cuántas horas hay que descansar (criterio negativo). En cuanto a la jornada diaria, la regla general es que el trabajador no puede trabajar más de nueve horas al día (criterio positivo $)^{4}$, teniendo que mediar al menos un descanso de doce horas no retribuidas entre jornada diaria y jornada diaria de trabajo (criterio negativo) ${ }^{5}$. En cuanto a la jornada semanal, la regla general es que «será de cuarenta horas semanales de trabajo efectivo de promedio en cómputo anual» (criterio positivo) ${ }^{6}$, teniendo en cuenta que «los trabajadores tendrán derecho a un descanso [retribuido] mínimo semanal ... de día y medio ininterrumpido que, como regla general, comprenderá la tarde del sábado o, en su

\footnotetext{
${ }^{1}$ Aprobado por Real Decreto Legislativo 1/1995, de 24 marzo. Sobre él y sus enmiendas, véase J. MARTÍNEZ GIRÓN y A. ARUFE VARELA, Derecho crítico del Trabajo. Critical Labor Law, 3 ed., Atelier (Barcelona, 2014), pp. 22 y ss.

${ }^{2}$ Asunto C-210/09.

${ }^{3}$ Aprobado por la Ley 7/2007, de 12 abril.

${ }^{4}$ Cfr. artículo 34, apartado 3, párrafo segundo, del Estatuto de los Trabajadores.

${ }^{5}$ Ibidem, párrafo primero.

${ }^{6}$ Cfr. artículo 34, apartado 1, párrafo segundo, del Estatuto de los Trabajadores.
} 
caso, la mañana del lunes y el día completo del domingo» (criterio negativo) ${ }^{7}$. En cuanto a la jornada anual, la legislación española de seguridad social (pero no el Estatuto de los Trabajadores), a propósito de la cotización a la seguridad social del trabajo a tiempo parcial, la cuantifica en 1.826 horas anuales (criterio positivo) ${ }^{8}$, teniendo en cuenta que el trabajador tiene derecho en cada año natural a catorce días festivos retribuidos ${ }^{9} \mathrm{y}$, además, a vacaciones anuales retribuidas, cuya duración nunca «será inferior a treinta días naturales» (criterio negativo) ${ }^{10}$.

3. El principio tradicional que preside la regulación contenida en los artículos 34 a 38 del Estatuto de los Trabajadores es el de que la jornada legal de trabajo es una condición de trabajo mínima. De un lado, esto significa que son nulos de pleno Derecho los convenios colectivos o los contratos individuales de trabajo que impongan jornadas diarias, semanales o anuales superiores a las máximas establecidas por dichos preceptos estatutarios $^{11}$. De otro lado, significa igualmente que los convenios colectivos y los contratos individuales de trabajo pueden establecer jornadas diarias, semanales o anuales más favorables para el trabajador que las reguladas en dichos preceptos estatutarios $^{12}$. En España, es muy frecuente la mejora colectiva del descanso semanal legal de día y medio, que suele ampliarse a dos días completos a la semana (sábado y domingo), pues la regla general en casi todos los sectores productivos es la semana laboral inglesa (ocho horas al día, de lunes a viernes). Pero los empresarios españoles consideran, sobre todo desde 1994, que esta regulación legal de la jornada de trabajo es muy rígida. En línea con el Derecho de la Unión Europea, exigen una mayor flexibilidad para poder aplicar las jornadas legales de trabajo. Este nuevo principio económico de la flexibilización de la jornada de trabajo es un tópico de moda en España, especialmente desde que se produjo el «crack» de Lehman Brothers. Según los empresarios, la clave está en la posibilidad de distribuir irregularmente la jornada de trabajo a lo largo del año, de manera que sólo existiría una única jornada de trabajo verdaderamente máxima, que es la jornada de trabajo anual (legal o, en su caso, convencional). A continuación, pasamos a examinar las medidas españolas de austeridad laboral relativas a la distribución irregular de la jornada de trabajo.

\section{APLICACIÓN PRÁCTICA}

4. En España, hasta el año 2010, el marco jurídico regulador de la jornada de trabajo estaba concebido para que pudiese existir una distribución irregular de la jornada semanal ordinaria de 40 horas a lo largo del año. Teóricamente, por ejemplo, eran posibles semanas laborales de 63 horas (esto es, 9 horas al día, de lunes a domingo), que se compensarían con otras semanas de sólo 17 horas, fundamentalmente por dos razones. En primer lugar, porque las 40 horas de la jornada ordinaria máxima legal eran

\footnotetext{
${ }^{7}$ Cfr. artículo 37, apartado 1, párrafo primero, del Estatuto de los Trabajadores.

${ }^{8}$ Cfr. disposición adicional séptima de la Ley General de Seguridad Social, aprobada por Real Decreto Legislativo 1/1994, de 20 junio. Sobre esta Ley, véase J. MARTíNEZ GIRÓN, A. ARUFE VARELA y X.M. CARRIL VÁZQUEZ, Derecho de la Seguridad Social, $3^{\mathrm{a}}$ ed., Netbiblo (A Coruña, 2013), pp. 18 y Ss.

${ }^{9}$ Cfr. artículo 37, apartado 2, del Estatuto de los Trabajadores.

${ }^{10}$ Cfr. artículo 38, apartado 1, del Estatuto de los Trabajadores.

${ }^{11}$ Cfr. artículos 3, apartado 1, 9 y 85, apartado 1, del Estatuto de los Trabajadores.

${ }^{12} \mathrm{Cfr}$. artículo 3, apartado 3, del Estatuto de los Trabajadores.
} 
sólo - en línea con el Derecho de la Unión Europea - un «promedio» en cómputo anual. En segundo lugar, porque los dos días reales de descanso semanal podían acumularse - igualmente en línea con el Derecho de la Unión Europea- en períodos de cómputo que excediesen de la semana correspondiente a su disfrute ${ }^{13}$. Pero existía un límite para llevar a cabo esa distribución irregular de la jornada a lo largo del año. Este límite - siempre en línea con el Derecho de la Unión Europea- era la necesidad de que la distribución irregular de la jornada estuviese autorizada por el convenio colectivo aplicable en la empresa. Y según estudios patrocinados por la agencia estatal española encargada de fomentar la negociación colectiva (esto es, la Comisión Consultiva Nacional de Convenios Colectivos), aproximadamente la mitad de los convenios colectivos estipulados en España hasta el año 2007 no preveía la posibilidad de que las empresas cubiertas por ellos pudiesen acogerse a la posibilidad de distribuir irregularmente la jornada semanal a lo largo del año ${ }^{14}$.

5. A partir del año 2010 (por tanto, en plena crisis económico-financiera), el Gobierno central español pretendió acabar con esta tendencia de la negociación colectiva. A este efecto, el Real Decreto-ley 7/2011, de 10 junio, de medidas urgentes para la reforma de la negociación colectiva, promulgado a iniciativa del Presidente socialista del Gobierno, Sr. RODRÍGUEZ ZAPATERO, impuso (a título de «medidas para contribuir a la flexibilidad interna en la empresa») que todos los convenios colectivos que se negociasen deberían incluir «un porcentaje máximo y mínimo de la jornada de trabajo que podrá distribuirse de manera irregular a lo largo del año» ${ }^{15}$, teniendo en cuenta que «salvo pacto en contrario, este porcentaje será de un cinco por ciento ${ }^{16}$. Tras las elecciones generales de noviembre de 2011, el Sr. RODRÍGUEZ ZAPATERO fue sustituido como Presidente del Gobierno por el derechista Sr. Mariano RAJOY. En el marco de sus muchas medidas de austeridad laboral, el nuevo Gobierno español liberalizó más radicalmente la distribución irregular de la jornada a lo largo del año. Esta liberalización cristalizó en la Ley 3/2012, de 6 julio, de medidas urgentes para la reforma del mercado laboral. Esta Ley modificó el Estatuto de los Trabajadores en materia de jornada de trabajo. Según ella, «mediante convenio colectivo o, en su defecto, por acuerdo entre la empresa y los representantes de los trabajadores, se podrá establecer la distribución irregular de la jornada a lo largo del año» ${ }^{17}$, pero teniendo en cuenta - aquí esta la nueva liberalización — que «en defecto de pacto, la empresa podrá distribuir de manera irregular el diez por ciento de la jornada de trabajo» ${ }^{18}$.

6. En España, el control del cumplimiento de los preceptos legales y convencionales en materia de jornada está encomendado a la Inspección de Trabajo. Esta Inspección actúa aplicando el Real Decreto Legislativo 5/2000, de 4 agosto, que contiene el texto refundido de la Ley sobre infracciones y sanciones administrativas de naturaleza social. Según esta Ley, son «infracciones graves» del empresario «la transgresión de las normas y los límites legales o pactados en materia de jornada,

\footnotetext{
${ }^{13} \mathrm{Cfr}$. artículo 37, apartado 1, párrafo primero, del Estatuto de los Trabajadores.

${ }^{14}$ Véase J.M. GOERLICH PESET (Coordinador), El tiempo de trabajo en la negociación colectiva, Ministerio de Trabajo e Inmigración (Madrid, 2008), pp. 67 y ss.

${ }^{15}$ Cfr. artículo 2, apartado 1.

${ }^{16}$ Ibidem.

${ }^{17}$ Cfr. artículo 9, apartado 1.

${ }^{18}$ Ibidem.
} 
trabajo nocturno, horas extraordinarias, horas complementarias, descansos, vacaciones, permisos y, en general, el tiempo de trabajo a que se refieren los artículos ... 34 a 38 del Estatuto de los Trabajadores» ${ }^{19}$. Según esta misma Ley, estas infracciones se castigan con «multas» administrativas, teniendo en cuenta que las graves se sancionan «con multa, en su grado mínimo, de 626 a 1.250 euros; en su grado medio de 1.251 a 3.125 euros; y en su grado máximo de 3.126 a 6.250 euros $\gg{ }^{20}$. Estas sanciones administrativas pueden ser impugnadas ante los tribunales laborales ${ }^{21}$. La Inspección de Trabajo considera difícil perseguir los incumplimientos legales del empresario en materia de jornada de trabajo. Ello se debe al hecho de que casi siempre le resulta posible al empresario compensar los excesos de jornada impuestos al trabajador mediante la concesión a este último de períodos de descanso compensatorio, como paradigmáticamente ocurre en materia de horas extraordinarias (según el Estatuto de los Trabajadores, «no se computarán las horas extraordinarias [cuyo tope máximo es ochenta horas al año] que hayan sido compensadas mediante descanso dentro de los cuatro meses siguientes a su realización») ${ }^{22}$.

\section{HECHOS Y CIFRAS}

7. Sobre la base de que en España existen convenios colectivos (empresariales y sectoriales) de ámbito geográfico provincial (en España, hay cincuenta provincias), de ámbito geográfico regional (en España, existen diecisiete regiones, llamadas Comunidades Autonómas) y de ámbito nacional, en el período comprendido entre julio y septiembre de 2014, se ha negociado un total veintiséis convenios colectivos de ámbito nacional ${ }^{23}$. Todos estos convenios colectivos nacionales han sido publicados en

\footnotetext{
${ }^{19}$ Cfr. artículo 7, apartado 5.

${ }^{20} \mathrm{Cfr}$. artículo 40, apartado 1, letra b).

${ }^{21}$ Cfr. artículo 2, letra n), de la Ley 36/2011, de 10 octubre, reguladora de la jurisdicción social.

${ }^{22}$ Cfr. artículo 35 , apartado 2, párrafo segundo.

${ }^{23}$ Trece de ellos en el mes de julio, esto es: 1) Convenio colectivo de Activa Innovación y Servicios, SAU
} (Boletín Oficial del Estado de 8 julio 2014); 2) II convenio colectivo de Anjana Investments, SLU (Boletín Oficial del Estado de 8 julio 2014); 3) II convenio colectivo para el personal de tierra de Ferrys Rápidos del Sur, SLU y Forde Reederei Seetouristik Ibéria, SLU (Boletín Oficial del Estado de 8 julio 2014); 4) Convenio colectivo de Aviva Grupo Corporativo, SL, Aviva Vida y Pensiones, SA, Aviva Gestión, S.G.I.I.C. y Aviva Servicios Compartidos, AIE (Boletín Oficial del Estado de 10 julio 2014); 5) Convenio colectivo de Bull (España), SA (Boletín Oficial del Estado de 17 julio 2014); 6) Convenio colectivo de Kiabi España Ksce, SA (Boletín Oficial del Estado de 17 julio 2014); 7) Convenio colectivo de Plus Ultra Seguros Generales y Vida, SA de Seguros y Reaseguros (Boletín Oficial del Estado de 17 julio 2014); 8) Convenio colectivo de Serviabertis, SL (Boletín Oficial del Estado de 17 julio 2014); 9) Convenio colectivo de Sociedad General de Autores y Editores (Boletín Oficial del Estado de 21 julio 2014); 10) Convenio colectivo de Air Nostrum LAM (Pilotos), SA (Boletín Oficial del Estado de 28 julio 2014); 11) Convenio colectivo de Capital Genetic EBT, SL (Boletín Oficial del Estado de 30 julio 2014); 12) III convenio colectivo de Easyjet Handling Spain, sucursal en España (Boletín Oficial del Estado de 30 julio 2014); y 13) XXVI convenio colectivo de Bimbo, SAU (Boletín Oficial del Estado de 30 julio 2014). Ocho de ellos en el mes de agosto, esto es: 1) II convenio colectivo de Agfa Graphics NV, sucursal en España (Boletín Oficial del Estado de 14 agosto 2014); 2) IX convenio colectivo de Siemens, SA (Boletín Oficial del Estado de 15 agosto 2014); 3) Convenio colectivo general para el sector de mantenimiento y conservación de instalaciones acuáticas (Boletín Oficial del Estado de 19 agosto 2014); 4) Convenio colectivo de Bio Ecdeorest, SL, Ecdeorest Geotermia, SL, Biomasa Ecdeorestal de Villacañas, SAU and Vapormatra, SA (Boletín Oficial del Estado de 21 agosto 2014); 5) III convenio colectivo de Orange Espagne, SAU (Boletín Oficial del Estado de 21 agosto 2014); 6) Convenio colectivo para la industria de alimentos compuestos para animales (Boletín Oficial del Estado de 21 August 2014); 7) Convenio colectivo de Mapfre Grupo Asegurador (Boletín Oficial del Estado de 21 agosto 2014); and 8) VII convenio colectivo de BP Oil España, SAU (Boletín Oficial del Estado de 22 agosto 2014). Y 
el Boletín Oficial del Estado, durante el trimestre correspondiente, cabiendo su utilización gratuita y a texto completo en el sitio oficial en Internet de dicho Boletín, ubicado en www.boe.es. Dos de esos veintiséis convenios colectivos de ámbito nacional son convenios colectivos sectoriales ${ }^{24}$. Los veinticuatro convenios colectivos restantes son convenios colectivos empresariales de ámbito geográfico nacional, aunque cuatro de ellos se refieren a grupos de empresas ${ }^{25}$, y otro más a un concreto oficio existente en una empresa de ámbito nacional ${ }^{26}$. Centrando nuestra atención en esta muestra —que consideramos significativa - de veintiséis convenios colectivos muy recientes, hemos pretendido comprobar hasta qué punto se ha realizado o no la intención de los sucesivos Gobiernos centrales españoles, a que antes aludimos, de modificar las tendencias de la negociación colectiva en materia de distribución irregular de la jornada de trabajo, incrementando el tratamiento de este tópico en los convenios colectivos negociados últimamente en nuestro país.

8. Tomando esta muestra de veintiséis convenios colectivos de ámbito nacional en su conjunto, resulta aparentemente que las tendencias existentes antes de las reformas operadas en 2010 en el Estatuto de los Trabajadores no han cambiado. En efecto, quince de esos convenios colectivos regulan la distribución irregular de la jornada de trabajo a lo largo del año (en consecuencia, un 57,6 por ciento del total ${ }^{27}$,

cinco de ellos en el mes de septiembre, esto es: 1) Convenio colectivo de Control y Montajes Industriales CYMI, SA (Boletín Oficial del Estado de 5 septiembre 2014); 2) Convenio colectivo para los trabajadores de servicios auxiliares de ISS Facility Services, SA (Boletín Oficial del Estado de 12 septiembre 2014); 3) Convenio colectivo de Pastificio Service, SL (Boletín Oficial del Estado de 12 septiembre 2014); 4) Convenio colectivo de Asociación para la Gestión de la Integración Social (Boletín Oficial del Estado de 13 septiembre 2014); and 5) Convenio colectivo de AP-1 Europistas, Concesionaria del Estado, SAU (Boletín Oficial del Estado de 13 septiembre 2014).

${ }^{24}$ Esto es, convenio colectivo general para el sector de mantenimiento y conservación de instalaciones acuáticas (Boletín Oficial del Estado de 19 agosto 2014), y el convenio colectivo para la industria de alimentos compuestos para animales (Boletín Oficial del Estado de 21 agosto 2014).

${ }^{25}$ Esto es, II convenio colectivo para el personal de tierra de Ferrys Rápidos del Sur, SLU y Forde Reederei Seetouristik Ibéria, SLU (Boletín Oficial del Estado de 8 julio 2014), convenio colectivo de Aviva Grupo Corporativo, SL, Aviva Vida y Pensiones, SA, Aviva Gestión, S.G.I.I.C. y Aviva Servicios Compartidos, AIE (Boletín Oficial del Estado de 10 julio 2014), convenio colectivo de Bio Ecdeorest, SL, Ecdeorest Geotermia, SL, Biomasa Ecdeorestal de Villacañas, SAU y Vapormatra, SA (Boletín Oficial del Estado de 21 agosto 2014), y convenio colectivo de Mapfre Grupo Asegurador (Boletín Oficial del Estado de 21 agosto 2014).

${ }^{26}$ Esto es, convenio colectivo de Air Nostrum LAM (Pilotos), SA (Boletín Oficial del Estado de 28 julio 2014).

${ }^{27}$ Se trata de los siguientes: 1) Convenio colectivo de Activa Innovación y Servicios, SAU (Boletín Oficial del Estado de 8 julio 2014); 2) II convenio colectivo de Anjana Investments, SLU (Boletín Oficial del Estado de 8 julio 2014); 3) Convenio colectivo de Bull (España), SA (Boletín Oficial del Estado de 17 julio 2014); 4) Convenio colectivo de Kiabi España Ksce, SA (Boletín Oficial del Estado de 17 julio 2014); 5) XXVI convenio colectivo de Bimbo, SAU (Boletín Oficial del Estado de 30 julio 2014); 6) IX convenio colectivo de Siemens, SA (Boletín Oficial del Estado de 15 agosto 2014); 7) Convenio colectivo general para el sector de mantenimiento y conservación de instalaciones acuáticas (Boletín Oficial del Estado de 19 agosto 2014); 8) Convenio colectivo de Bio Ecdeorest, SL, Ecdeorest Geotermia, SL, Biomasa Ecdeorestal de Villacañas, SAU y Vapormatra, SA (Boletín Oficial del Estado de 21 agosto 2014); 9) Convenio colectivo para la industria de alimentos compuestos para animales (Boletín Oficial del Estado de 21 agosto 2014); 10) Convenio colectivo de Mapfre Grupo Asegurador (Boletín Oficial del Estado de 21 agosto 2014); 11) VII convenio colectivo de BP Oil España, SAU (Boletín Oficial del Estado de 22 agosto 2014); 12) Convenio colectivo para los trabajadores de servicios auxiliares de ISS Facility Services, SA (Boletín Oficial del Estado de 12 septiembre 2014); 13) Convenio colectivo de Pastificio Service, SL (Boletín Oficial del Estado de 12 septiembre 2014); 14) Convenio colectivo de Asociación para la Gestión de la Integración Social (Boletín Oficial del Estado de 13 septiembre 2014); y 
mientras que los once restantes prescinden de regular dicho tópico (en consecuencia, un $42,4$ por ciento del total $)^{28}$. Pero si se examina la muestra desde un punto de vista funcional, resulta que el cien por cien de los convenios colectivos sectoriales de ámbito nacional regula el tópico, mientras que en el caso de los convenios colectivos empresariales de ámbito nacional, el 54,1 por ciento aborda la regulación de la distribución irregular de la jornada en la correspondiente empresa o grupos de empresas de que se trate, mientras que el 45,9 por ciento omite de tratar dicha figura (dentro de este último porcentaje se incluye el único convenio colectivo de oficio contenido en la muestra). Son unos porcentajes que nos parecen lógicos. De un lado, porque a nivel de sector, ante el dilema flexibilidad o rigidez, resulta lógico que los negociadores de los correspondientes convenios colectivos, que pueden afectar a muchos cientos de empresas distintas, se pronuncien a favor de la flexibilidad en abstracto. De otro lado, en cuanto a los convenios colectivos de ámbito empresarial, porque no todas las empresas son iguales $\mathrm{y}$, en consecuencia, no todas tienen por qué necesitar la distribución irregular de la jornada a lo largo del año. Examinaremos a continuación los quince convenios colectivos de nuestra muestra, en los que aparece expresamente regulado el tópico en cuestión.

9. De entre estos quince convenios colectivos en los que se autoriza la distribución irregular de la jornada a lo largo del año, seis contienen una regulación muy genérica, orientada a posibilitar sin mayores matices la utilización de la institución durante el período de vigencia del convenio colectivo ${ }^{29}$. Los nueve convenios colectivos restantes (en consecuencia, un 60 por ciento del total) contienen una regulación mucho más concreta, que incluso impone límites al número de horas que pueden distribuirse irregularmente a lo largo del año ${ }^{30}$. Estos límites o topes son extraordinariamente

15) Convenio colectivo de AP-1 Europistas, Concesionaria del Estado, SAU (Boletín Oficial del Estado de 13 septiembre 2014).

${ }^{28}$ Se trata de los siguientes: 1) II convenio colectivo para el personal de tierra de Ferrys Rápidos del Sur, SLU y Forde Reederei Seetouristik Ibéria, SLU (Boletín Oficial del Estado de 8 julio 2014); 2) Convenio colectivo de Aviva Grupo Corporativo, SL, Aviva Vida y Pensiones, SA, Aviva Gestión, S.G.I.I.C. y Aviva Servicios Compartidos, AIE (Boletín Oficial del Estado de 10 julio 2014); 3) Convenio colectivo de Plus Ultra Seguros Generales y Vida, SA de Seguros y Reaseguros (Boletín Oficial del Estado de 17 julio 2014); 4) Convenio colectivo de Serviabertis, SL (Boletín Oficial del Estado de 17 julio 2014); 5) Convenio colectivo de Sociedad General de Autores y Editores (Boletín Oficial del Estado de 21 julio 2014); 6) Convenio colectivo de Air Nostrum LAM (Pilotos), SA (Boletín Oficial del Estado de 28 julio 2014); 7) Convenio colectivo de Capital Genetic EBT, SL (Boletín Oficial del Estado de 30 julio 2014); 8) III convenio colectivo de Easyjet Handling Spain, sucursal en España (Boletín Oficial del Estado de 30 julio 2014); 9) II convenio colectivo de Agfa Graphics NV, sucursal en España (Boletín Oficial del Estado de 14 agosto 2014); 10) III convenio colectivo de Orange Espagne, SAU (Boletín Oficial del Estado de 21 agosto 2014); and 11) Convenio colectivo de Control y Montajes Industriales CYMI, SA (Boletín Oficial del Estado de 5 septiembre 2014).

${ }^{29}$ Se trata de los siguientes: 1) Convenio colectivo de Activa Innovación y Servicios, SAU (Boletín Oficial del Estado de 8 julio 2014); 2) Convenio colectivo de Kiabi España Ksce, SA (Boletín Oficial del Estado de 17 julio 2014); 3) XXVI convenio colectivo de Bimbo, SAU (Boletín Oficial del Estado de 30 julio 2014); 4) Convenio colectivo de Mapfre Grupo Asegurador (Boletín Oficial del Estado de 21 agosto 2014); 5) Convenio colectivo de Pastificio Service, SL (Boletín Oficial del Estado de 12 septiembre 2014); and 6) Convenio colectivo de Asociación para la Gestión de la Integración Social (Boletín Oficial del Estado de 13 septiembre 2014).

${ }^{30}$ Se trata de los siguientes: 1) Convenio colectivo de Bull (España), SA (Boletín Oficial del Estado de 17 julio 2014); 2) II convenio colectivo de Anjana Investments, SLU (Boletín Oficial del Estado de 8 julio 2014); 3) IX convenio colectivo de Siemens, SA (Boletín Oficial del Estado de 15 agosto 2014); 4) Convenio colectivo general para el sector de mantenimiento y conservación de instalaciones acuáticas (Boletín Oficial del Estado de 19 agosto 2014); 5) Convenio colectivo de Bio Ecdeorest, SL, Ecdeorest 
variados, y parecen no tomar como referente el límite del 10 por ciento establecido por la legislación, en el caso de que no se hubiese producido acuerdo. Así, en ese conjunto de nueve convenios colectivos hemos encontrado los topes del 1,7 por ciento ${ }^{31}$, del 5 por ciento $^{32}$, del 5,5 por ciento $^{33}$, del 6 por ciento $^{34}$, del 8 por ciento ${ }^{35}$, del 10 por ciento $^{36}$ y del 15 por ciento $^{37}$, referidos siempre a las horas anuales de trabajo. En ocasiones, alguno de estos nueve convenios colectivos cuantifica incluso cómo pueden llegar a ser las jornadas laborales, si es que se aplica a las mismas la distribución irregular de la jornada máxima anual, afirmando — sobre la base de la jornada semanal legal de cuarenta horas a la semana, y sobre la base de que esta jornada puede promediarse a lo largo del año- que «la duración de la jornada irregular será de 48 horas máximas y 32 horas mínimas de trabajo a la semana, respetando el cómputo anual pactado» ${ }^{38}$.

\section{PROSPECTIVA}

10. Últimamente, la legislación laboral española posee una extraordinaria inestabilidad. Desde mayo de 2010 (esto es, cuando se constató oficialmente la existencia en España de la crisis económico-financiera), el Estatuto de los Trabajadores de 1995 ha sufrido hasta el momento presente más de veinte modificaciones. Todas estas modificaciones, incluidas las relativas a la regulación de la jornada de trabajo, se enmarcan en la realización del objetivo de la austeridad laboral. Al Presidente del Gobierno socialista, Sr. RODRÍGUEZ ZAPATERO, sus medidas de austeridad laboral le costaron perder las elecciones generales en noviembre de 2011. El actual Presidente del Gobierno español, Sr. Mariano RAJOY, ganó por mayoría absoluta esas elecciones generales. Pero sus incesantes medidas de austeridad laboral han creado un gran descontento en España, haciendo crecer partidos políticos en los que confluyen las aspiraciones de los ciudadanos españoles y trabajadores españoles, que están «indignados». En España, volverá a haber elecciones generales dentro de sólo un año, en noviembre de 2015. En todo el sur de Europa, parece ser una ley de cumplimiento

Geotermia, SL, Biomasa Ecdeorestal de Villacañas, SAU y Vapormatra, SA (Boletín Oficial del Estado de 21 agosto 2014); 6) Convenio colectivo para la industria de alimentos compuestos para animales (Boletín Oficial del Estado de 21 agosto 2014); 7) VII convenio colectivo de BP Oil España, SAU (Boletín Oficial del Estado de 22 agosto 2014); 8) Convenio colectivo para los trabajadores de servicios auxiliares de ISS Facility Services, SA (Boletín Oficial del Estado de 12 septiembre 2014); y 9) Convenio colectivo de AP-1 Europistas, Concesionaria del Estado, SAU (Boletín Oficial del Estado de 13 septiembre 2014).

${ }^{31} \mathrm{Cfr}$. convenio colectivo para la industria de alimentos compuestos para animales (Boletín Oficial del Estado de 21 agosto 2014), artículo 10.

${ }^{32}$ Cfr. convenio colectivo de Bio Ecdeorest, SL, Ecdeorest Geotermia, SL, Biomasa Ecdeorestal de Villacañas, SAU y Vapormatra, SA (Boletín Oficial del Estado de 21 agosto 2014), artículo 16.

${ }^{33}$ Cfr. convenio colectivo para el sector de mantenimiento y conservación de instalaciones acuáticas (Boletín Oficial del Estado de 19 agosto 2014), artículo 40.

${ }^{34}$ Cfr. VII convenio colectivo de BP Oil España, SAU (Boletín Oficial del Estado de 22 agosto 2014), artículo 63 .

${ }^{35}$ Cfr. convenio colectivo de AP-1 Europistas, Concesionaria del Estado, SAU (Boletín Oficial del Estado de 13 septiembre 2014), artículo XI.

${ }^{36}$ Cfr. convenio colectivo de Bull (España), SA (Boletín Oficial del Estado de 17 julio 2014), artículo 24.1.

${ }^{37}$ Cfr. convenio colectivo para los trabajadores de servicios auxiliares de ISS Facility Services, SA (Boletín Oficial del Estado de 12 septiembre 2014), artículo 20.

${ }^{38}$ Cfr. IX convenio colectivo de Siemens, SA (Boletín Oficial del Estado de 15 agosto 2014), artículo 21. 
inexorable (parecida a la ley de la gravedad y a otras leyes de la naturaleza física) la de que el partido gobernante que recorta acaba perdiendo las elecciones y deja de gobernar. Si esta ley se cumple en España, con toda probabilidad el nuevo Gobierno español procederá a enmendar otra vez el Estatuto de los Trabajadores de 1995. Si esto sucede, veremos con toda probabilidad una nueva regulación en materia de jornada de trabajo. Pero esto es sólo un futurible. Habrá que esperar a ver qué ocurre. Esta incertidumbre concluirá, por estas mismas fechas, el año que viene.

\section{BIBLIOGRAFÍA}

MARTÍNEZ GIRÓN, Jesús; A. ARUFE VARELA, Alberto, Derecho crítico del Trabajo. Critical Labor Law, 3 ed., Atelier, Barcelona, 2014.

MARTÍNEZ GIRÓN, Jesús; A. ARUFE VARELA, Alberto; CARRIL VÁZQUEZ, Xosé Manuel, Derecho de la Seguridad Social, $3^{\text {a }}$ ed., Netbiblo, A Coruña, 2013.

GOERLICH PESET, José María (coord.), El tiempo de trabajo en la negociación colectiva, Ministerio de Trabajo e Inmigración, Madrid, 2008. 